\title{
Relato de Caso Clínico: Atendimento Pré Hospitalar (APH) a Hemorragia Exsanguinante relacionada a Trauma Crânio Encefálico (TCE)
}

\author{
Clinical Case Report: Pre-Hospital Care (PHC) to Exsanguinating Hemorrhage Related to Cranio \\ Encephalic Trauma (TBI)
}

\author{
Wagner Ferreira dos Santos ${ }^{1}$, Tássia Rayane de Arruda ${ }^{2}$, Juliana Vasconcelos da Silva² ${ }^{2}$ Mirella \\ Figueirôa de Alencar ${ }^{2}$, Jeniffa Priscila de Farias Santos ${ }^{3}$
}

\begin{abstract}
${ }^{1}$ Graduado em Enfermagem, Teresina, Piauí, Brasil. ${ }^{2}$ Graduanda em Enfermagem, Recife, Pernambuco, Brasil. ${ }^{3}$ Bacharel em Enfermagem, Vitória de Santo Antão, Pernambuco, Brasil. *Autor para correspondência. E-mail: wagnedopiaui@gmail.com

Resumo: Introdução: Os traumas inabituais são considerados acidentes que os serviços de assistência médica preferem nomear de lesões traumáticas aleatórias e inevitáveis. Relato de caso: Unidade de Suporte Básico (USB) foi acionada às $17 \mathrm{~h} 00$ para atender a um paciente do sexo masculino, aproximadamente 30 anos, com lesão na cabeça, com sinais de embriaguez, vítima de queda da própria altura. Na ambulância básica tripulava o técnico em enfermagem socorrista acompanhando a Residente (R1) de Atendimento PréHospitalar (APH) e o condutor socorrista. Discussão: O traumatismo crânio encefálico é uma agressão de causa externa que causa lesões que envolve o couro cabeludo do crânio, encéfalo, sistema nervoso central e um processo que pode durar dias ou semanas, mas começa logo no momento do impacto, com uma combinação de danos neurológicos, insuficiência vasculares efeitos inflamatórios.
\end{abstract}

Palavras-chave: Atendimento Pré Hospitalar (APH), Hemorragia Exsanguinante, Trauma Crânio Encefálico (TCE).

\begin{abstract}
Introduction: Unusual traumas are considered accidents that health care services prefer to name random and unavoidable traumatic injuries. Case Report: Basic Support Unit (BSSU) was called at 5:00 pm to attend to a male patient, approximately 30 years old, with head injury, with signs of intoxication, victim of falling from his own height. The basic ambulance was manned by the nursing technician and the firstaid nurse, accompanied by the Pre-Hospital Care (PHC) Resident (R1) and the first-aid driver. Discussion: Traumatic brain injury is an aggression of external cause that causes injuries involving the skull scalp, brain, central nervous system and a process that can last for days or weeks, but begins right at the moment of impact, with a combination of neurological damage, vascular insufficiency inflammatory effects.
\end{abstract}

Keywords: Pre-hospital care (PHC), Exsanguinating haemorrhage, Traumatic Brain Injury (TBI).

\section{Introdução}

Anualmente, cerca de 5,8 milhões de pessoas morrem por trauma em todo o mundo, $32 \%$ a mais que a soma das mortes por malária, AIDS e tuberculose. A mortalidade por trauma corresponde a $10 \%$ de todas as causas de morte e, sem as devidas intervenções, prevê-se que esta proporção aumentará até 2030. Além disso, os traumas também respondem pela maioria de incapacitações permanentes. A maior parte dos traumas ocorre entre pessoas de 5 a 44 anos, ou seja, crianças, jovens e adultos jovens. O Traumatismo Crânio Encefálico (Ceccon, Guetter, \& Pimentel, 2019) está associado a altas taxas de mortalidade e pode levar a sequelas graves que impactam na qualidade de vida. Um trabalho de revisão sobre TCE realizado no Brasil mostrou a grande importância de uma avaliação neurológica inicial a fim de se evitar óbitos e sequelas no caso de traumas graves (Ceccon et al., 2019).

Os dados epidemiológicos revelam que adolescentes e adultos jovens, seguidos por indivíduos com idade acima de 65 anos, são as populações mais atingidas pelas Lesões Cerebrais Traumáticas (LCTs) (Klauber et al., 1981).

Visto que o traumatismo cranioencefálico é a causa mais comum de invalidez de longo prazo e morte entre adultos jovens, ele representa um enorme fardo socioeconômico e de saúde. Como consequência da lesão primária, desenvolve-se um edema cerebral perifocal, causando uma elevação da pressão intracraniana devido ao espaço intracraniano limitado. Isso acarreta uma redução da pressão de perfusão cerebral e do fluxo 
sanguíneo cerebral. Um déficit de perfusão cerebral abaixo do limiar para isquemia leva a novas lesões isquêmicas e a uma progressão da contusão (Ceccon et al., 2019).

O trauma no Brasil é a $3^{\text {a }}$ maior causa de morte entre os cidadãos brasileiros conforme dados do ministério da saúde. Considerando a relevância desse tema e os números alarmantes já citados anteriormente, o presente estudo de caso objetiva descrever o relato de um paciente de meia idade, alcoolizado, usuário de drogas que teve uma queda da própria altura e sofreu um TCE grave na região occipito-parietal com lesão corto-contusa de aproximadamente $6 \mathrm{~cm}$ com hemorragia arterial em sinais clínicos de choque. A hemorragia maciça é comum e frequentemente associada a alta morbidade e mortalidade (Ceccon et al., 2019).

Diante do supracitado, a metodologia utilizada neste estudo foi a descrição de um Relato de Caso referente a um trauma atendido pela Unidade de Suporte Básico (USB) do Serviço de Atendimento Móvel de Urgência (SAMU) na capital do Estado de Pernambuco. O objetivo deste relato de caso é descrever a experiência da equipe de enfermagem frente ao atendimento do paciente vítima de trauma crânio encefálico com base em protocolos adotados no atendimento pré-hospitalar que visam à melhoria na morbimortalidade desse perfil de paciente/trauma.

\section{Materiais e métodos}

Trata-se de um relato de estudo de caso de um paciente atendido na Unidade de Suporte Básico (USB). Os dados obtidos no Atendimento Pré- Hospitalar (APH) foram registrados no prontuário do paciente. O trabalho se baseou em uma Revisão Integrativa, com uma avaliação criteriosa de artigos científicos, investigando o tema proposto. É importante salientar que o estudo foi autorizado.

Segundo com Souza et al. (2010) Revisão Integrativa inclui análises de pesquisas que direcionam a prática clínica, possibilitando assim o conhecimento sobre assuntos, preenchendo as lacunas de outros estudos. Para avaliar os artigos coletados foi feita a leitura, em seguida o fichamento dos artigos que serão retomados para compor a discussão. A Revisão integrativa a qual se baseia esse trabalho é uma ferramenta que tem sido importante para área da saúde, tendo em vista que possibilita a síntese dos conhecimentos referentes à temática pretendida.

Ainda que seja um delineamento de pesquisa desafiador, pois necessita de uma abordagem rigorosa ao campo da enfermagem, no sentido que analisa os dados de maneira eficiente, baseado em evidências, o que torna o campo da enfermagem em qualitativo.

Para área da Enfermagem esse método é fundamental, pois através da leitura resumida é possível estabelecer discussões sobre os temas. As etapas da revisão integrativa consistem 5 etapas. A primeira onde ocorre o estabelecimento da hipótese por meio da escolha e definição do tema, objetivos e identificação das palavras chaves (Souza et al., 2010).

Na segunda etapa foi feita a amostragem, ou busca de literatura, estabelecendo os critérios de exclusão e inclusão. Na terceira etapa categorizaram-se os estudos, organizando as informações. Na quarta etapa avaliaram-se os estudos incluídos na revisão aplicando as análises estatísticas.

$\mathrm{Na}$ quinta etapa interpretaram-se os dados e se discutiu os resultados, propondo recomendações e sugestões futuras. As pesquisas para esse trabalho foram realizadas nas bibliotecas virtuais de saúde, além das bases de dados MEDLINE-Medical Literature Analysis; LILACS, Literatura Latino-Americana e do Caribe SciELO-Scientific Electronic Library Online.

\section{Fundamentação Teórica}

Na década de 1980, as causas externas passaram a representar a segunda causa de morte no Brasil. Esse crescimento estaria ligado a fatores diversos, como sociais, econômicos, políticos e tecnológicos, configurando como um problema de saúde pública, em razão da mortalidade, custos e vidas perdidas, o que gera impacto na família e na sociedade (Ramos \& Jardim, 2004). As vítimas dessas causas externas, normalmente ficam com sequelas de traumatismo cranioencefálico, cujos óbitos ocorrem em três picos: em segundos ou minutos após lesão provocados da aorta, coração medula e tronco cerebral; ou em algumas horas após o trauma decorrente de hemorragias e lesão do sistema nervoso central e o terceiro após 24 horas, em decorrência da falência de múltiplos órgãos e infecção (Ramos \& Jardim, 2004).

O traumatismo crânio encefálico e uma agressão de causa externa que causa lesões que envolve o couro cabeludo do crânio, encéfalo, sistema nervoso central e um processo que pode durar dias ou semanas, mas começa logo no momento do impacto, com uma combinação de danos neurológicos, insuficiência vasculares efeitos inflamatórios. Esse tipo de trauma ocorre após lesões fechadas ou penetrante as estruturas 
encefálicas. Os tipos de lesões crânio encefálico incluem concussão, contusão, fratura do crânio, hematoma epidural ou subidural, hemorragia subaracnoide e herniação (Ramos \& Jardim, 2004).

O traumatismo crânio encefálico e um grande problema de saúde pública no âmbito mundial, o TCE está cada vez mais incidente no mundo moderno. Foi a partir dessas análises que essa relevância patológica que a causa maior e por acidentes e atos de violência, a evolução do homem e de suas tecnologias. (Barbosa et al., 2006). Entre as causas principais de TCE, e possível citar os acidentes automobilísticos, na grande maioria dos casos, seguindo dos atropelamentos, acidente ciclísticos e moto ciclísticos, agressões físicas, quedas e lesões por armas de fogo, entre outros menos frequentes, podendo resultar em graves sequelas ou em lesões cranianas relativamente pequenas (Ramos \& Jardim, 2004).

A função da equipe de enfermagem e muito importante no cuidado oferecido a vítima de TCE, havendo necessidades de maior aptidão para realização de anamnese por meio de coleta e história da vítima, realizar exames físicos, coletas e exames e intervir através de tratamento imediato, enfatizando a manutenção da vida (Ramos \& Jardim, 2004).

O enfermeiro se sobressai por seus atributos peculiares de cuidado e organização de uma assistência diminuída de riscos, que lhe garante a abordagem inicial na classificação no setor de emergência/urgência, diagnosticar, realizar encaminhamentos, controlar a demanda de atendimento e coordenar os demais membros da equipe, o que pode ser percebido nos apontamentos que assinala que a Sistematização da assistência em enfermagem garante uma ampla autonomia para o enfermeiro, um respaldo seguro e significativo através do registro de diagnósticos e intervenções, que permite a continuidade/complementaridade multiprofissional, além de estabelecer uma aproximação enfermeiro paciente enfermeiro - equipe multiprofissional. Sendo ainda inegável um conjunto de conhecimentos e habilidades que preparem o enfermeiro a um atendimento humanizado (Vieira, 2016).

O TCE ocasiona impacto na saúde da população em geral com influência na morbidade e mortalidade do paciente, com cerca de $15 \%$ a $20 \%$ das mortes em vítimas com idade entre 5 e 35 anos, sendo responsável por 1\% de todos os óbitos em adultos (Vieira, 2016).

A gravidade do TCE é classificada de acordo com a Escala de Coma de Glasgow (GCS), cujos parâmetros são obtidos mediante abertura ocular, resposta verbal e resposta motora. Essa escala foi descrita em 1974 por Teasdale e Jennet, que categorizaram o TCE em grave uma pontuação de 3 a 8, moderado 9 a 12 e como leve 13 a 15. Ainda que os testes e evidências sobre o TCE e suas sequelas neurológicas sejam cada vez mais reais, a população ainda não leva a sério, pois, apenas $60 \%$ dos pacientes que sobrevivem a TCE, ficam com deficiências motoras e cognitivas (Monteiro et al., 2016).

Quando o paciente é admitido nas unidades hospitalares, o enfermeiro deverá obter conhecimento de sua história no Atendimento Pré-Hospitalar, além de imobilizar a coluna cervical e realizar aspiração orotraquela para manutenção da oxigenação, possibilitando ao paciente uma ventilação adequada e mantendo a cabeça inclinada. Além observar o decúbito, manter o acesso venoso calibroso ou cateter central para volemia, fazendo o balanço hídrico em cada hora (Vieira, 2016).

Os agravos encéfalo-cerebrais no TCE se definem pelo impacto e pelas lesões penetrantes que se classificam como difusas, focais, primárias e secundárias, sendo que podem estar associados em um mesmo paciente, mas, com prevalência de um dos tipos. As difusas podem ocorrer sobre a forma de agravo axonal difuso e hemorragias que agridem o cérebro e provocam rotação do crânio, axônios e vasos, ocasionando isquemia e tumefação (Moura, 2016).

A lesão focal epidural é constituída por uma aglomeração de sangue na face do crânio, com incidência na região temporal em decorrência da fratura, com perda de consciência transitória, seguido de desordem e coma. Já as lesões primárias resultam do choque exercido sobre a caixa craniana devido o processo inflamatório das células do sistema nervoso, onde é necessária a monitoração da pressão intracraniana (Oliveira, 2011).

No manuseio do paciente com TCE, o enfermeiro utiliza protocolos que possuem o objetivo de garantir uma avaliação e prontidão dinâmica nas intervenções e estabilização das condições respiratórias, ventilatórias e hemodinâmicas da vítima, o que possibilita maior eficácia na assistência. Por esse motivo o enfermeiro deverá possuir conhecimento científico sobre o TCE para que possa avaliar a vítima com habilidade, precisão e segurança (Almeida \& Brasileiro, 2018).

Dessa forma, é preciso repensar a forma da assistência prestada as vítimas do TCE, de maneira acolhedora, técnica e humanizada. Também é importante enfatizar que as políticas públicas de saúde devem elaborar estratégias preventivas em relação as causas externas, como acidentes de veículos, drogas, bebidas alcoólicas, pois muitas das lesões do traumatismo de cranioencefálico ocorrem por esses fatores (Leite, 2018). No primeiro instante da assistência ao indivíduo vítima de traumatismo, é essencial uma apreciação minuciosa para a tomada de decisões acertadas, realizando uma visão integral das condições clínicas do paciente, como 
nível de consciência, sinais vitais (SSVV), sinais e sintomas inerentes às possíveis sequelas decorrentes do trauma e identificando precocemente os eventos que podem comprometer a vida. Com relação a esses parâmetros, proceder às medidas de emergência cabíveis para adequar os padrões clínicos e a remoção do paciente à unidade de suporte avançado. $\mathrm{O}$ atendimento a essas vítimas requer da equipe de enfermagem um atendimento sincronizado e rápido com técnicas e habilidades. $O$ enfermeiro se sobressai nos cuidados da TCE, por seus atributos peculiares de cuidado e organização de uma assistência diminuída.

\section{Relato do Estudo de Caso}

Unidade de Suporte Básico (USB) foi acionada às $17 \mathrm{~h} 00$ para atender a um paciente do sexo masculino, aproximadamente 30 anos, com lesão na cabeça, com sinais de embriaguez, vítima de queda da própria altura. $\mathrm{Na}$ ambulância básica tripulava o técnico em enfermagem socorrista acompanhando a Residente (R1) de Atendimento Pré-Hospitalar (APH) e o condutor socorrista. Ao chegar ao local, ambiente sem sinais de perigo eminente, foi encontrado o paciente sentado com hemorragia considerável na região craniana, perda sanguínea significativa já com presença de sangue coagulado na face e roupa do mesmo e chão. Foi constatado segundo informações colhidas com curiosos que esse paciente estava com esse trauma há aproximadamente uma hora e meia.

Foi realizado o protocolo XABCDE do trauma, segundo o Pré hospital Trauma Life Support (PHTLS), foi realizado o curativo compressivo no local da hemorragia que se encontrava na região occipito-parietal com um ferimento corto-contuso de aproximadamente 6 centímetros, apresentando hemorragia arterial. Em seguida, realizou-se a avaliação de vias aéreas com controle da coluna cervical, verificação da simetria de tórax e avaliação ventilatória que evidenciou uma taquipneia. Ao exame neurológico: Paciente com Glasgow de 15 porém apresentando sonolência. Realizada transferência adequada do paciente para a viatura, realizado acesso venoso periférico em membro superior esquerdo na veia cefálica com cateter intravenoso calibroso (Jelco de $\mathrm{n}^{\circ} 16$ ) para viabilizar hidratação com ringer lactato, avaliação pupilar evidenciando pupilas isocóricas e fotorreagentes.

Quanto aos sinais vitais (SSVV): PA: 120/80 mmHg FC:125 BPM SpO2: 97\% FR: 20 RPM ECG: 15/15. Segundo a $9^{a}$ edição do PHTLS Na pesquisa primária de um paciente com trauma, com risco eminente a vida e hemorragia externa, esta deve ser imediatamente identificada e gerenciada. Se a hemorragia externa exsanguinante estiver presente, deve ser controlada antes mesmo da avaliação da via aérea (ou simultaneamente, se a assistência adequada estiver presente na cena) ou realização de outras intervenções, como a imobilização da coluna cervical.

\section{Conclusão}

O chamado TCE- Traumatismo Crânio Encefálico é ocasionado por fatores físicos no crânio, geralmente por um impacto externo que pode ser penetrante com desenvolvimento de transformações cerebrais. Essas modificações surgidas do impacto trazem incapacidade intelectual, transtornos de mobilidade momentâneas ou irreversíveis. Essa pesquisa e de grande importância para aprimorar o conhecimento em relação a vítima de TCE. O Traumatismo Crânio Encefálico pode ser classificado como leve, moderado e grave. No manuseio com o paciente que sofre TCE, o profissional de enfermagem faz uso de procedimentos que garantem avaliação precisa para intervenções e estabilização das condições respiratórias, ventilatórias e hemodinâmicas, o que facilita eficiência no atendimento.

O Atendimento Pré-Hospitalar a essas vítimas requer da equipe de enfermagem sincronia e rapidez com técnicas e habilidades, dando ênfase ao enfermeiro, multiplicidades de conhecimentos e compreensão da liderança da equipe de enfermagem. Por isso é muito importante que toda equipe de enfermagem que atua na urgência e emergência, tenho curso profissionalizantes e constante atualização dos conhecimentos teóricos e práticos para melhor atender a vítima de trauma. Os estudos apontam que a condução do atendimento a esses pacientes, ponderam as sequelas, a partir de um acompanhamento mais humanizado e com a sistematização de cuidados na equipe multiprofissional.

\section{Referências}

Ceccon, A., Guetter, C. R., \& Pimentel, S. K. 2019. Avaliação de pacientes vítimas de trauma cranioencefálico com sinais de intoxicação alcoólica. Revista do Colégio Brasileiro de Cirurgiões, 46(5).

Klauber, M. R., Barrett-Connor, E., Marshall, L. F., \& Bowers, S. A. 1981. The epidemiology of head injury: a prospective study of an entire community-San Diego County, California, 1978. American journal of epidemiology, 113(5), 500-509. 
Ramos, A., \& Jardim, S. R. 2004. Lesão cerebral traumática e acidentes de trabalho: estudo de revisão. Revista Brasileira de Saúde Ocupacional, 29, 27-32.

Barbosa, F. T., Cunha, R. M. D., Rocha, A. P. C., \& Silva Júnior, H. J. L. 2006. Intraventricular pneumocephalus after accidental perforation of the dura mater: Case report. Revista brasileira de anestesiologia, 56, 511-517.

Almeida, L. C. F., \& Brasileiro, M. E. 2018. Atuação do Enfermeiro no Atendimento ao Paciente com Traumatismo Crânioencefálico: Revisão Bibliográfica. Revista Científica Multidisciplinar Núcleo do Conhecimento, 3, 139-148.

Leite, R. A. 2018. Assistência de enfermagem prestada a vítima de trauma crânio-encefálico: uma análise da literatura. In Congresso Brasileiro de Ciências da Saúde.

Monteiro, L. F., Frasson, M. Z., Wrsesinski, A., Bardini, A. V. L. S., Lin, J., \& Fernandes, A. F. 2016. Caracterização dos pacientes com traumatismo cranioencefálico grave admitidos em um hospital terciário. Arquivos Catarinenses de Medicina, 45(3), 2-16.

Moura, A. D. M. 2016. Atuação do enfermeiro emergencista no atendimento pré-hospitalar ao paciente com traumatismo crânio- encefálico (TCE). In Simpósio de TCC e Seminário de IC.

Oliveira, L. D. A. M., da Cunha Soares, Y. K., Noleto, L. C., Fontinele, A. V. C., Galvão, M. P. S. P., \& de Souza, J. M. 2018. Assistência de enfermagem em pacientes vítimas de traumatismo crânio encefálico: revisão integrativa. Revista uningá, 55(2), 33-46.

Souza, M. T. D., Silva, M. D. D., \& Carvalho, R. D. 2010. Revisão integrativa: o que é e como fazer. Einstein, 8 , 102-106.

Vieira, R. S. 2016. Intervenções de enfermagem ao paciente com traumatismo crânio encefálico: revisão integrativa. In XX Enfermaio I Mostra do internato em enfermagem. Fortaleza - CE.

\section{Minicurrículo}

Wagner Ferreira dos Santos. Técnico em enfermagem; bacharelado em enfermagem, FAPI (Faculdade do Piauí). Treinamento em atendimento pré-hospitalar promovido pelo núcleo de educação permanente do SAMU Recife-PE, urgências respiratórias e ventilação mecânica atendimento pré-hospitalar ofertado pela Emergência, Enfermagem Obstétrica, Atendimento pré-hospitalar a queimados.

Tássia Rayane de Arruda. Graduada em Enfermagem pela Universidade Estácio do Recife (2018); pósgraduada em Enfermagem Psiquiátrica e Saúde Mental (2021), pós-graduanda em Enfermagem em Urgência e Emergência e Enfermagem em UTI (2021).

Juliana Vasconcelos da Silva. Enfermeira formada na Universidade Salgado de Oliveira Monitora de Legislação e Ética de Enfermagem Pós GraduandA em Urgência/Emergência e UTI

Mirella Figueirôa de Alencar. Graduanda do $8^{\circ}$ período de Enfermagem. Trabalhou como Técnica de Enfermagem no Hospital Maria Lucinda - HML , no Serviço de Atendimento Domiciliar - SAD da Prefeitura do Recife, sediado no HML e como Socorrista do Serviço de Atendimento Móvel de Urgência - SAMU Recife. Atualmente, Técnica de Enfermagem Estatuária do Governo de Pernambuco em Hospital Correia Picanço na Unidade de Terapia Intensiva de Doenças Infecto Parasitarias - UTI/DIP.

Jeniffa Priscila de Farias Santos. Graduanda do $8^{\circ}$ período de Enfermagem. Trabalhou como Técnica de Enfermagem no Hospital Maria Lucinda - HML , no Serviço de Atendimento Domiciliar - SAD da Prefeitura do Recife, sediado no HML e como Socorrista do Serviço de Atendimento Móvel de Urgência - SAMU Recife. Atualmente, Técnica de Enfermagem Estatuária do Governo de Pernambuco em Hospital Correia Picanço na Unidade de Terapia Intensiva de Doenças Infecto Parasitarias - UTI/DIP. 
Como citar: Santos, W.F., Arruda, T.R., Silva, J.V., Alencar, M.F., \& Santos, J.P.F. 2021. Relato de Caso Clínico: Atendimento Pré Hospitalar (APH) a Hemorragia Exsanguinante relacionada a Trauma Crânio Encefálico (TCE). Pubsaúde, 7, a235. DOI: https://dx.doi.org/10.31533/pubsaude7.a235

Recebido: 3 ago. 2021.

Revisado e aceito: 24 ago. 2021.

Conflito de interesse: os autores declaram, em relação aos produtos e companhias descritos nesse artigo, não ter interesses associativos, comerciais, de propriedade ou financeiros que representem conflito de interesse.

Licenciamento: Este artigo é publicado na modalidade Acesso Aberto sob a licença Creative Commons Atribuição 4.0 (CC-BY 4.0). 